\title{
Radiating Gravitational Collapse with an Initial Inhomogeneous Energy Density Distribution
}

\author{
Pinheiro, G. $1 *$ and R. Chan $2+$ \\ 1 Divisão de Programas de Pós-Graduação ${ }^{2}$ Coordenação de Astronomia e Astrofísica, \\ Observatório Nacional, Rua General José Cristino, 77, \\ São Cristóvão 20921-400, Rio de Janeiro, RJ, Brazil
}

(Dated: 18/11/2010)

\begin{abstract}
A new model is proposed to a collapsing star consisting of an initial inhomogeneous energy density and anisotropic pressure fluid with shear, radial heat flow and outgoing radiation. In previous papers one of us has always assumed an initial star with homogeneous energy density. The aim of this work is to generalize the previous models by introducing an initial inhomogeneous energy density and compare it to the initial homogeneous energy density collapse model. We will show the differences between these models in the evolution of all physical quantities that characterizes the gravitational collapse. The behavior of the energy density, pressure, mass, luminosity and the effective adiabatic index is analyzed. The pressure of the star, at the beginning of the collapse, is isotropic but due to the presence of the shear the pressure becomes more and more anisotropic. The black hole is never formed because the apparent horizon formation condition is never satisfied, in contrast of the previous model where a black hole is formed. An observer at infinity sees a radial point source radiating exponentially until reaches the time of maximum luminosity and suddenly the star turns off. In contrast of the former model where the luminosity also increases exponentially, reaching a maximum and after it decreases until the formation of the black hole. The effective adiabatic index is always positive without any discontinuity in contrast of the former model where there is a discontinuity around the time of maximum luminosity. The collapse is about three thousand times slower than in the case where the energy density is initially homogeneous.

PACS numbers:
\end{abstract}

*Electronic address: gpinheiro@on.br

${ }^{\dagger}$ Electronic address: chan@on.br 
Keywords: Gravitational Collapse, Shear, Inhomogeneous Energy Density, Black Hole, General Relativity

\section{INTRODUCTION}

One of the most outstanding problems in gravitation theory is the evolution of a collapsing massive star, after it has exhausted its nuclear fuel. The problem of constructing physically realistic models for radiating collapsing stars is one of the aims of the relativistic astrophysics. However, in order to obtain realistic models we need to solve complicated systems of nonlinear differential equations. In many cases we can simplify the problem considering some restrictions in these equations and solve the system analytically. Such models, although simplified, are useful to construct simple exact solutions, which are at least physically reasonable. This allows a clearer analysis of the main physical effects at play, and it can be very useful for checking of numerical procedures.

The majority of the previous works have considered only shear-free motion of the fluid [10] 11 [17]. This simplification allows us to obtain exact solutions of the Einstein's equations in some cases but it is somewhat unrealistic. It is also unrealistic to consider heat flow without viscosity but if viscosity is introduced, it is desirable to allow shear in the fluid motion, although we have not included the viscosity in this work. Thus, it is interesting to study solutions that contains shear, because it plays a very important role in the study of gravitational collapse, as shown in [4 8, 19 21] and in [22]. More recent studies on this subject with and without shear are also found in [24]-[28].

In the first paper [4] [5] we have compared two collapsing model: a shear-free and a shearing model. We were interested in studying the effect of the shearing motion in the evolution of the collapse. It was shown that the pressure of the star, at the beginning of the collapse, is isotropic but due to the presence of the shear the pressure becomes more and more anisotropic. The anisotropy in self-gravitating systems has been reviewed and discussed the causes for its appearance in [11]. As shown by [4] [5] the simplest cause of the presence of anisotropy in a self-gravitating body is the shearing motion of the fluid, because it appears without an imposition ad-hoc [3].

In the second paper [7] we have studied a model of a collapsing radiating star consisting of an anisotropic fluid with initial homogeneous energy density and shear viscosity undergoing 
radial heat flow with outgoing radiation, but without bulk viscosity.

In the third paper [8] we have analyzed a model of a collapsing radiating star consisting of an anisotropic fluid with initial homogeneous energy density and bulk viscosity undergoing radial heat flow with outgoing radiation, but without shear viscosity.

The aim of this work is to generalize our previous models by introducing initial inhomogeneous energy density, but without viscosity.

This work is organized as follows. In Section 2 we present the Einstein's field equations. In Section 3 we derive the junction conditions. In Section 4 we present the proposed solution of the field equations. In Section 5 we describe the model considered in this work for the initial configuration. In Section 6 we present the energy conditions for an initial inhomogeneous energy density fluid. In Section 7 we show the time evolution of the total mass, luminosity, the effective adiabatic index and in Section 8 we summarize the main results obtained in this work.

\section{FIELD EQUATIONS}

We assume a spherically symmetric distribution of fluid undergoing dissipation in the form of heat flow. While the dissipative fluid collapses it produces radiation. The interior spacetime is described by the most general spherically symmetric metric, using comoving coordinates,

$$
d s_{-}^{2}=-A^{2}(r, t) d t^{2}+B^{2}(r, t) d r^{2}+C^{2}(r, t)\left(d \theta^{2}+\sin ^{2} \theta d \phi^{2}\right) .
$$

The exterior spacetime is described by Vaidya's [16] metric, which represents an outgoing radial flux of radiation,

$$
d s_{+}^{2}=-\left[1-\frac{2 m(v)}{\mathbf{r}}\right] d v^{2}-2 d v d \mathbf{r}+\mathbf{r}^{2}\left(d \theta^{2}+\sin ^{2} \theta d \phi^{2}\right),
$$

where $m(v)$ represents the mass of the system inside the boundary surface $\Sigma$, function of the retarded time $v$.

We assume the interior energy-momentum tensor is given by 


$$
\begin{aligned}
G_{\alpha \beta}= & \kappa T_{\alpha \beta}=\kappa\left[\left(\mu+p_{t}\right) u_{\alpha} u_{\beta}+p_{t} g_{\alpha \beta}+\left(p-p_{t}\right) X_{\alpha} X_{\beta}+\right. \\
& \left.+q_{\alpha} u_{\beta}+q_{\beta} u_{\alpha}\right]
\end{aligned}
$$

where $\mu$ is the energy density of the fluid, $p$ is the radial pressure, $p_{t}$ is the tangential pressure, $q^{\alpha}$ is the radial heat flux, $X_{\alpha}$ is an unit four-vector along the radial direction, $u^{\alpha}$ is the four-velocity, which have to satisfy $u^{\alpha} q_{\alpha}=0, X_{\alpha} X^{\alpha}=1, X_{\alpha} u^{\alpha}=0$ and $\kappa=8 \pi$ (i.e., $c=G=1)$.

The shearing tensor $\sigma_{\alpha \beta}$ is defined as

$$
\sigma_{\alpha \beta}=u_{(\alpha ; \beta)}+\dot{u}_{(\alpha} u_{\beta)}-\frac{1}{3} \Theta\left(g_{\alpha \beta}+u_{\alpha} u_{\beta}\right)
$$

with

$$
\begin{gathered}
\dot{u}_{\alpha}=u_{\alpha ; \beta} u^{\beta}, \\
\Theta=u_{; \alpha}^{\alpha},
\end{gathered}
$$

where $\Theta$ is the expansion scalar, the semicolon denotes a covariant derivative and the parentheses in the indices mean symmetrizations.

Since we utilize comoving coordinates we have,

$$
u^{\alpha}=A^{-1} \delta_{0}^{\alpha},
$$

and since the heat flux is radial

$$
q^{\alpha}=q \delta_{1}^{\alpha}
$$

Thus the non-zero components of the shearing tensor are given by

$$
\begin{gathered}
\sigma_{11}=2 \frac{B^{2}}{3 A}\left(\frac{\dot{B}}{B}-\frac{\dot{C}}{C}\right), \\
\sigma_{22}=-\frac{C^{2}}{3 A}\left(\frac{\dot{B}}{B}-\frac{\dot{C}}{C}\right), \\
\sigma_{33}=\sigma_{22} \sin ^{2} \theta .
\end{gathered}
$$

A simple calculation shows that

$$
\sigma_{\alpha \beta} \sigma^{\alpha \beta}=\frac{2}{3 A^{2}}\left(\frac{\dot{B}}{B}-\frac{\dot{C}}{C}\right)^{2}
$$


Thus, if we define the scalar $\sigma$ as

$$
\sigma=-\frac{1}{3 A}\left(\frac{\dot{B}}{B}-\frac{\dot{C}}{C}\right),
$$

we can write that

$$
\begin{gathered}
\sigma_{11}=-2 B^{2} \sigma, \\
\sigma_{22}=C^{2} \sigma, \\
\sigma_{33}=C^{2} \sigma \sin ^{2} \theta .
\end{gathered}
$$

Using (1) and (6), we can write that

$$
\Theta=\frac{1}{A}\left(\frac{\dot{B}}{B}+2 \frac{\dot{C}}{C}\right) .
$$

The non-vanishing components of the field equations, using (11), (3), (17), (8) and (6), interior of the boundary surface $\Sigma$ are

$$
\begin{gathered}
G_{00}^{-}=-\left(\frac{A}{B}\right)^{2}\left[2 \frac{C^{\prime \prime}}{C}+\left(\frac{C^{\prime}}{C}\right)^{2}-2 \frac{C^{\prime}}{C} \frac{B^{\prime}}{B}\right]+ \\
+\left(\frac{A}{C}\right)^{2}+\frac{\dot{C}}{C}\left(\frac{\dot{C}}{C}+2 \frac{\dot{B}}{B}\right)=\kappa A^{2} \mu \\
G_{11}^{-}=\frac{C^{\prime}}{C}\left(\frac{C^{\prime}}{C}+2 \frac{A^{\prime}}{A}\right)-\left(\frac{B}{C}\right)^{2}- \\
-\left(\frac{B}{A}\right)^{2}\left[2 \frac{\ddot{C}}{C}+\left(\frac{\dot{C}}{C}\right)^{2}-2 \frac{\dot{A}}{A} \frac{\dot{C}}{C}\right] \\
=\kappa B^{2} p, \\
G_{22}^{-}=\left(\frac{C}{B}\right)^{2}\left[\frac{C^{\prime \prime}}{C}+\frac{A^{\prime \prime}}{A}+\frac{C^{\prime}}{C} \frac{A^{\prime}}{A}-\frac{A^{\prime}}{A} \frac{B^{\prime}}{B}-\frac{B^{\prime}}{B} \frac{C^{\prime}}{C}\right]+ \\
+\left(\frac{C}{A}\right)^{2}\left[-\frac{\ddot{B}}{B}-\frac{\ddot{C}}{C}-\frac{\dot{C}}{C} \frac{\dot{B}}{B}+\frac{\dot{A}}{A} \frac{\dot{C}}{C}+\frac{\dot{A}}{A} \frac{\dot{B}}{B}\right] \\
=\kappa C^{2} p_{t},
\end{gathered}
$$




$$
\begin{gathered}
G_{33}^{-}=G_{22}^{-} \sin ^{2} \theta \\
G_{01}^{-}=-2 \frac{\dot{C}^{\prime}}{C}+2 \frac{C^{\prime}}{C} \frac{\dot{B}}{B}+2 \frac{A^{\prime}}{A} \frac{\dot{C}}{C}=-\kappa A B^{2} q .
\end{gathered}
$$

The dot and the prime stand for differentiation with respect to $t$ and $r$, respectively.

\section{JUNCTION CONDITIONS}

We consider a spherical surface with its motion described by a time-like three-space $\Sigma$, which divides spacetimes into interior and exterior manifolds. For the junction conditions we follow the approach given by [12] [13]. Hence we have to demand

$$
\begin{gathered}
\left(d s_{-}^{2}\right)_{\Sigma}=\left(d s_{+}^{2}\right)_{\Sigma}, \\
K_{i j}^{-}=K_{i j}^{+},
\end{gathered}
$$

where $K_{i j}^{ \pm}$is the extrinsic curvature to $\Sigma$, given by

$$
K_{i j}^{ \pm}=-n_{\alpha}^{ \pm} \frac{\partial^{2} x_{ \pm}^{\alpha}}{\partial \xi^{i} \partial \xi^{j}}-n_{\alpha}^{ \pm} \Gamma_{\beta \gamma}^{\alpha} \frac{\partial x_{ \pm}^{\beta}}{\partial \xi^{i}} \frac{\partial x_{ \pm}^{\gamma}}{\partial \xi^{j}},
$$

and where $\Gamma_{\beta \gamma}^{\alpha}$ are the Christoffel symbols, $n_{\alpha}^{ \pm}$the unit normal vectors to $\Sigma, x^{\alpha}$ are the coordinates of interior and exterior spacetimes and $\xi^{i}$ are the coordinates that define the surface $\Sigma$.

From the junction conditions (23) and (24) we obtain the following results (see more details in [4]-[8])

$$
m=\left\{\frac{C}{2}\left[1+\left(\frac{\dot{C}}{A}\right)^{2}-\left(\frac{C^{\prime}}{B}\right)^{2}\right]\right\}_{\Sigma},
$$

which is the total energy entrapped inside the surface $\Sigma[2]$,

$$
p=(q B)_{\Sigma},
$$


(This result is analogous to the one obtained by [4] [5] [7] [8] for a shearing fluid motion.)

$$
L_{\infty}=\frac{\kappa}{2}\left[p C^{2}\left(\frac{C^{\prime}}{B}+\frac{\dot{C}}{A}\right)^{2}\right]_{\Sigma},
$$

which is the total luminosity for an observer at rest at infinity and

$$
1+z_{\Sigma}=\left(\frac{C^{\prime}}{B}+\frac{\dot{C}}{A}\right)_{\Sigma}^{-1}
$$

which is the boundary redshift $z_{\Sigma}$. The boundary redshift can be used to determine the time of formation of the horizon.

\section{SOLUTION OF THE FIELD EQUATIONS}

As in [4] [5] [7] [8] we are proposing solutions of the field equations (18)-(22) with the form

$$
\begin{gathered}
A(r, t)=A_{0}(r), \\
B(r, t)=B_{0}(r), \\
C(r, t)=r B_{0}(r) f(t),
\end{gathered}
$$

where $A_{0}(r)$ and $B_{0}(r)$ are solutions of a static perfect fluid having $\mu_{0}$ as the energy density and $p_{0}$ as the isotropic pressure.

We have chosen this separation of variables in the metric functions, in order to have the following properties: when $f(t) \rightarrow 1$ the metric functions represent the static solution of the initial star configuration and the collapse takes place when $f(t) \rightarrow 0$. We stress here that, following the junction condition, equation (23), the function $C\left(r_{\Sigma}, t\right)$ represents the luminosity radius of the body as seen by an exterior observer.

Thus, the expansion scalar (17) can be written as

$$
\Theta=\frac{2}{A_{0}}\left(\frac{\dot{f}}{f}\right)
$$


and the shear scalar (13) can be written as

$$
\sigma=\frac{1}{3 A_{0}}\left(\frac{\dot{f}}{f}\right)
$$

Now the equations (18)-(22) can be written as

$$
\begin{gathered}
\kappa \mu=\kappa \mu_{0}+\frac{1}{A_{0}^{2}}\left(\frac{\dot{f}}{f}\right)^{2}+\frac{1}{r^{2} B_{0}^{2}}\left(\frac{1}{f^{2}}-1\right) \\
\kappa p=\kappa p_{0}-\frac{1}{A_{0}^{2}}\left[2 \frac{\ddot{f}}{f}+\left(\frac{\dot{f}}{f}\right)^{2}\right]-\frac{1}{r^{2} B_{0}^{2}}\left(\frac{1}{f^{2}}-1\right), \\
\kappa p_{t}=\kappa p_{0}-\frac{1}{A_{0}^{2}}\left(\frac{\ddot{f}}{f}\right) \\
\kappa q=\frac{2}{A_{0} B_{0}^{2}}\left[\left(\frac{\dot{f}}{f}\right)\left(\frac{B_{0}^{\prime}}{B_{0}}+\frac{1}{r}-\frac{A_{0}^{\prime}}{A_{0}}\right)\right],
\end{gathered}
$$

where

$$
\begin{gathered}
\kappa \mu_{0}=-\frac{1}{B_{0}^{2}}\left[2 \frac{B_{0}^{\prime \prime}}{B_{0}}-\left(\frac{B_{0}^{\prime}}{B_{0}}\right)^{2}+\frac{4}{r} \frac{B_{0}^{\prime}}{B_{0}}\right], \\
\kappa p_{0}=\frac{1}{B_{0}^{2}}\left[\left(\frac{B_{0}^{\prime}}{B_{0}}\right)^{2}+\frac{2}{r} \frac{B_{0}^{\prime}}{B_{0}}+2 \frac{A_{0}^{\prime}}{A_{0}} \frac{B_{0}^{\prime}}{B_{0}}+\frac{2}{r} \frac{A_{0}^{\prime}}{A_{0}}\right] .
\end{gathered}
$$

We can see from equations (35)-(38) that when the function $f(t)=1$ we obtain the static perfect fluid configuration.

Substituting equations (36), (38) and (33)-(34) into (27), assuming also that $p_{0}\left(r_{\Sigma}\right)=0$, we obtain a second order differential equation in $f(t)$,

$$
2 \frac{\ddot{f}}{f}+\left(\frac{\dot{f}}{f}\right)^{2}+a\left(\frac{\dot{f}}{f}\right)+b\left(\frac{1}{f^{2}}-1\right)=0
$$

where

$$
a=\left[2\left(\frac{A_{0}}{B_{0}}\right)\left(\frac{B_{0}^{\prime}}{B_{0}}+\frac{1}{r}-\frac{A_{0}^{\prime}}{A_{0}}\right)\right]_{\Sigma},
$$



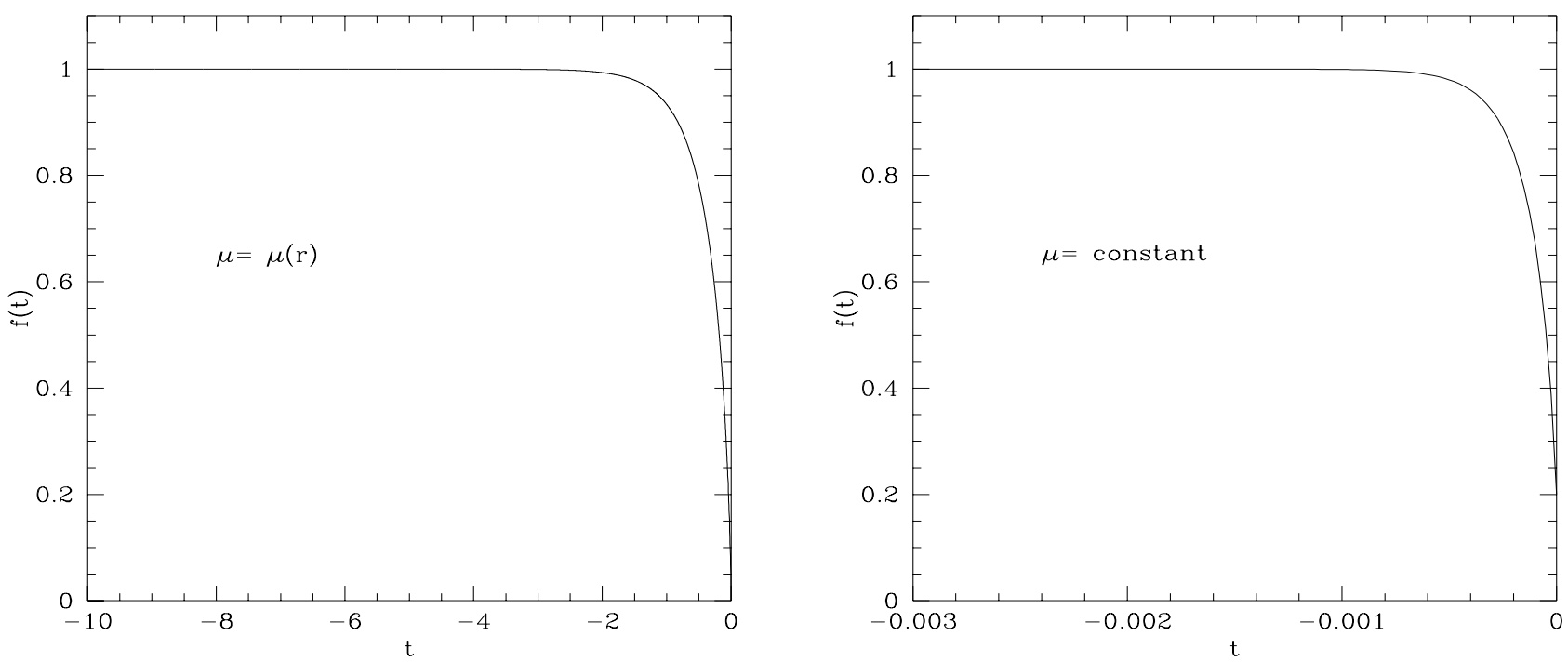

FIG. 1: Time behavior of the function $f(t)$ for the models with homogeneous and initial inhomogeneous density. The time is in units of second and $f(t)$ is dimensionless. The collapse for the initial homogeneous energy density model $(\mu=$ constant $)$ is 3000 times faster than that one for the initial inhomogeneous energy density model $(\mu=\mu(r))$.

and

$$
b=\left(\frac{A_{0}^{2}}{r^{2} B_{0}^{2}}\right)_{\Sigma} .
$$

Now, we can write equation (41) in the following way

$$
2 f \ddot{f}+\dot{f}^{2}+a f \dot{f}+b\left(1-f^{2}\right)=0,
$$

This equation is identical to the one obtained in [4] [5] [7] [8]. Thus, as before it has to be solved numerically (figure 1), assuming that at $t \rightarrow-\infty$ represents the static configuration with $\dot{f}(t \rightarrow-\infty) \rightarrow 0$ and $f(t \rightarrow-\infty) \rightarrow 1$. We also assume that $f(t \rightarrow 0) \rightarrow 0$. This means that the luminosity radius $C\left(r_{\Sigma}, t\right)$ has the value $r_{\Sigma} B_{0}\left(r_{\Sigma}\right)$ at the beginning of the collapse and vanishing at the end of the evolution.

\section{MODEL OF THE INITIAL CONFIGURATION}

We consider that the system at the beginning of the collapse has a static configuration of a perfect fluid satisfying [29] 


$$
\begin{gathered}
A_{0}^{2}=d_{0}\left[\frac{1+(\alpha+\beta) g(r)}{1+(\alpha-\beta) g(r)}\right]^{1 / \beta}, \\
B_{0}^{2}=c_{0} \frac{g(r)^{2}}{a_{0}^{2}\left(1+2 \alpha g(r)+2 g(r)^{2}\right)}\left[\frac{1+(\alpha-\beta) g(r)}{1+(\alpha+\beta) g(r)}\right]^{(\alpha+1) / \beta},
\end{gathered}
$$

where

$$
\begin{gathered}
g(r)=\frac{a_{0}}{1-r^{2}} \\
\beta=\sqrt{\alpha^{2}-2}, \\
d_{0}=\left(\frac{2 r_{\Sigma}-m_{0}}{2 r_{\Sigma}+m_{0}}\right)^{2}\left[\frac{1+(\alpha-\beta) g\left(r_{\Sigma}\right)}{1+(\alpha+\beta) g\left(r_{\Sigma}\right)}\right]^{1 / \beta}, \\
c_{0}=\frac{a_{0}^{2}}{g\left(r_{\Sigma}\right)}\left(1+\frac{m_{0}}{2 r_{\Sigma}}\right)^{4}\left[1+2 \alpha g\left(r_{\Sigma}\right)+2 g\left(r_{\Sigma}\right)^{2}\right]\left[\frac{1+(\alpha+\beta) g\left(r_{\Sigma}\right)}{1+(\alpha-\beta) g\left(r_{\Sigma}\right)}\right]^{(\alpha+1) / \beta}, \\
a_{0}=\frac{2 r_{\Sigma}}{m_{0}}\left(1-r_{\Sigma}^{2}\right), \\
\frac{r_{\Sigma}^{3}}{m_{0}\left(1-r_{\Sigma}^{2}\right)}-\frac{m_{0}}{4 r_{\Sigma}\left(1-r_{\Sigma}^{2}\right)}-\frac{2 r_{\Sigma}}{m_{0}},
\end{gathered}
$$

and where $r_{\Sigma}$ is the initial coordinate radius of the star in comoving coordinates and $m_{0}$ is the initial mass of the system. Thus the static initial inhomogeneous energy density and static pressure are given by

$$
\begin{aligned}
\kappa \mu_{0}= & \frac{1}{B_{0}^{2}\left[\left(1-r^{2}\right)^{2}+2 \alpha a_{0}\left(1-r^{2}\right)+2 a_{0}^{2}\right]^{2}}\left[-4\left(2 r^{2}-3 a_{0}+1+4 \alpha a_{0}\right)\left(1-r^{2}\right)^{2}-\right. \\
& 8\left(1-r^{2}\right)^{3}-8\left(\alpha a_{0}-3 r^{2} a_{0}-3 \alpha a_{0}^{2}-r^{2} \alpha a_{0}+2 a_{0}^{2}\right)\left(1-r^{2}\right)- \\
& \left.4 a_{0}^{2}\left(2-6 a_{0}-4 \alpha r^{2}-5 r^{2}\right)\right] \\
\kappa p_{0}= & \frac{1}{B_{0}^{2}\left[\left(1-r^{2}\right)^{2}+2 \alpha a_{0}\left(1-r^{2}\right)+2 a_{0}^{2}\right]^{2}} \times \\
& \quad\left[4\left(1-r^{2}\right)^{3}+\left(4 r^{2}+8 \alpha a_{0}\right)\left(1-r^{2}\right)^{2}+8 a_{0}^{2}\left(1-r^{2}\right)-4 a_{0}^{2} r^{2}\right] .
\end{aligned}
$$

This solution shows that the pressure and the energy density are finite and positive everywhere inside the star, the pressure and energy density decreases toward the boundary and that the speed of sound is smaller than the speed of the light $c$ everywhere. 
We consider the initial configuration as due to an helium core of a presupernova with $m_{0}=$ $6 M_{\odot}$, with $r_{\Sigma}=2.1232 \times 10^{5} \mathrm{~km}$ which corresponds approximately the same geometrical radius $r_{\Sigma} B_{0}\left(r_{\Sigma}\right)=2.1218 \times 10^{5} \mathrm{~km}[18]$.

With these values we can solve numerically the differential equation (44). Since the equation (45) gives us positive or negative values for $A_{0}$, we have assumed that $A_{0}<0$. Thus, we can see from (38), using (45)-(50) and this initial configuration, that $\left[\left(B_{0}^{\prime} / B_{0}+\right.\right.$ $\left.\left.1 / r-A_{0}^{\prime} / A_{0}\right) / A_{0}\right]_{\Sigma}<0,\left(B_{0}^{\prime} / B_{0}+1 / r\right)_{\Sigma}>0$, and by the fact that $q_{\Sigma}>0$ then we conclude that $\dot{f}<0$.

In the figure 1 we can note that the collapse for the initial homogeneous energy density model ( $\mu$ = constant) is 3000 times faster than that one for the initial inhomogeneous energy density model $(\mu=\mu(r))$. This fact may be due to two reasons. Firstly, the geometrical radius of the both models are not the same. Secondly, the metric functions $A_{0}(r)$ and $B_{0}(r)$ are also different, in such way that in the present work we have an initial monotonically decreasing energy density profile. This issue is also discussed in previous works [30] [31] in the context of the Tolman mass. These works have shown that a negative energy density gradient increases the Tolman mass of the system, thus decreasing the total collapse time. This result seems to agree with our present work. Besides, since the initial inhomogeneous model collapses slower is also related to the fact that in the present model the system ejects all its mass (this result will be shown later below) reducing drastically the gravitationally force term, unlike the initially homogeneous case where only about $33 \%$ of the mass is radiated.

In figure 2 we show the time evolution of the shear scalar, for different radii, for the two models (initial inhomogeneous and homogeneous energy density).

In order to determine the time of formation of the horizon $f_{\mathrm{bh}}$, we use the equations (29) and (301)-(32) and write

$$
\frac{\dot{f}_{\mathrm{bh}}}{f_{\mathrm{bh}}}=-\left[\frac{A_{0}}{B_{0}}\left(\frac{B_{0}^{\prime}}{B_{0}}+\frac{1}{r}\right)\right]_{\Sigma} \approx+1.4140 .
$$

Using the numerical solution of $f(t)$ and equation (55), we can see from figure 3 that the horizon is never formed, because the function $\dot{f} / f$ never reaches the value +1.4140 . At the first sight this fact could be interpreted as the formation of a naked singularity. However, this is not the case as we will see below in the calculation of the total energy entrapped 

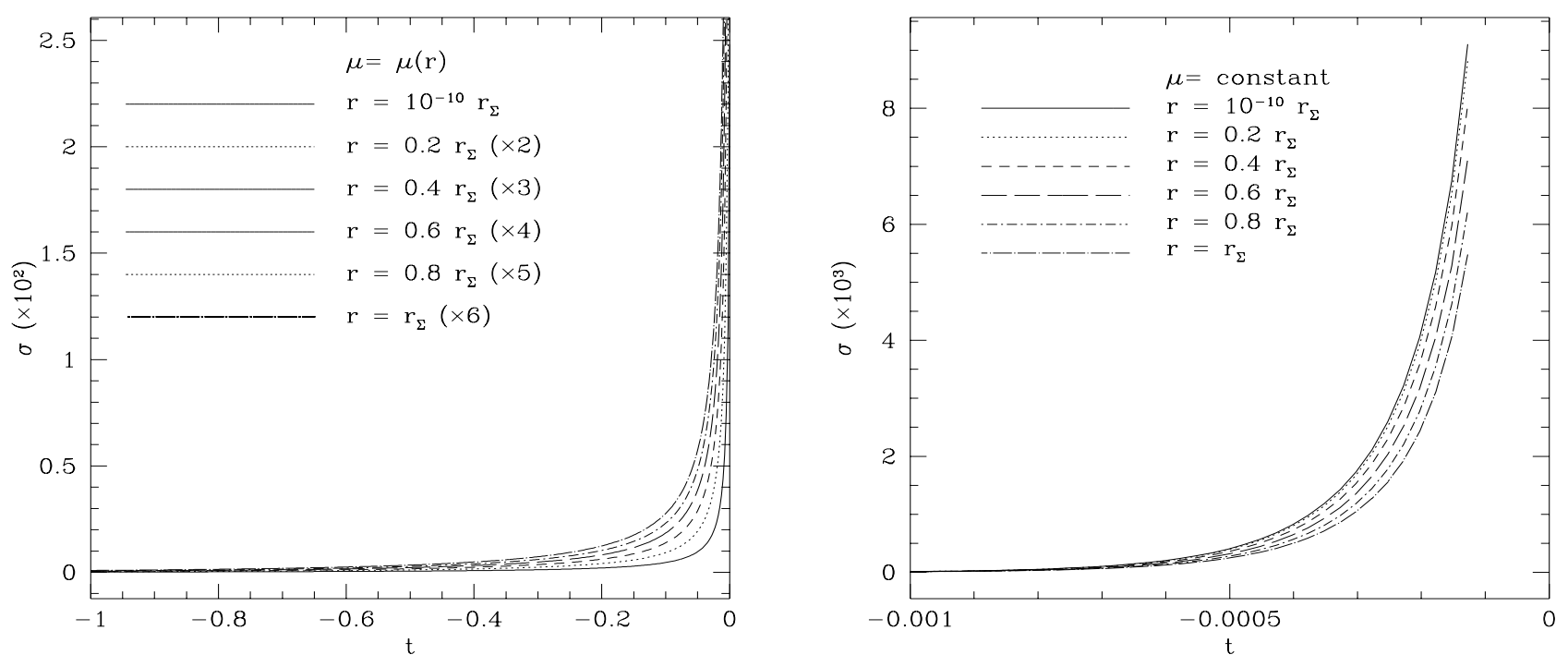

FIG. 2: Time evolution of the shear scalar at different radii for the initial inhomogeneous and homogeneous energy density model. The radii $r, r_{\Sigma}$ and the time are in units of seconds and the shear scalar is in units of $\sec ^{-1}$.

inside the hypersurface $\Sigma$.

It is shown in figures 4 and 8 the radial profiles of the energy density and the heat flux.

In figures 5 and 6 we plot the radial profiles of the radial and tangential pressures.

In figure 7 is shown the radial profiles of the radial and tangential pressure ratio. In this figure we can see that the star is isotropic at the beginning of the collapse $(f=1)$ but becoming more and more anisotropic at later times.

\section{ENERGY CONDITIONS FOR AN INITIAL INHOMOGENEOUS ENERGY DENSITY FLUID}

All known forms of matter obey the weak, dominant and strong energy conditions. For this reason a star model based on some fluid which violates these conditions cannot be seriously considered as physically relevant.

Thus, in order to find the energy conditions, we have followed the same procedure used in Kolassis, Santos \& Tsoubelis (1988) and have generalized the energy conditions for an anisotropic fluid. See more details in [7] [8]. These conditions are fulfilled if the following 

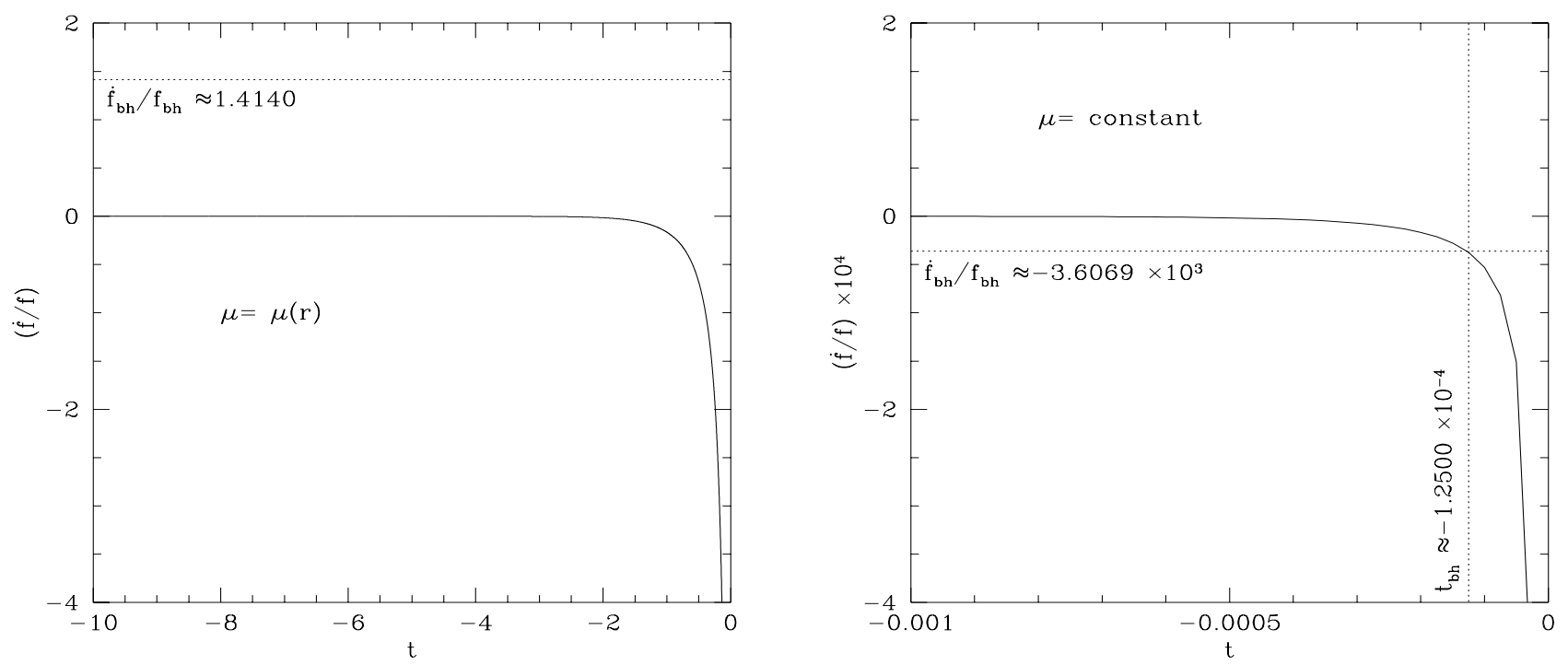

FIG. 3: The function $\dot{f} / f$ as a function of the time for the model with initial homogeneous and inhomogeneous energy density. The time is in units of second.

inequalities are satisfied:

$$
\begin{gathered}
\quad|\mu+p|-2|\bar{q}| \geq 0, \\
\text { (ii) } \quad \mu-p+2 p_{t}+\Delta \geq 0,
\end{gathered}
$$

and besides,

a) for the weak energy conditions

$$
\text { (iii) } \quad \mu-p+\Delta \geq 0
$$

b) for the dominant energy conditions

$$
\text { (iv) } \mu-p \geq 0
$$

$$
\text { (v) } \quad \mu-p-2 p_{t}+\Delta \geq 0
$$

c) for the strong energy conditions 

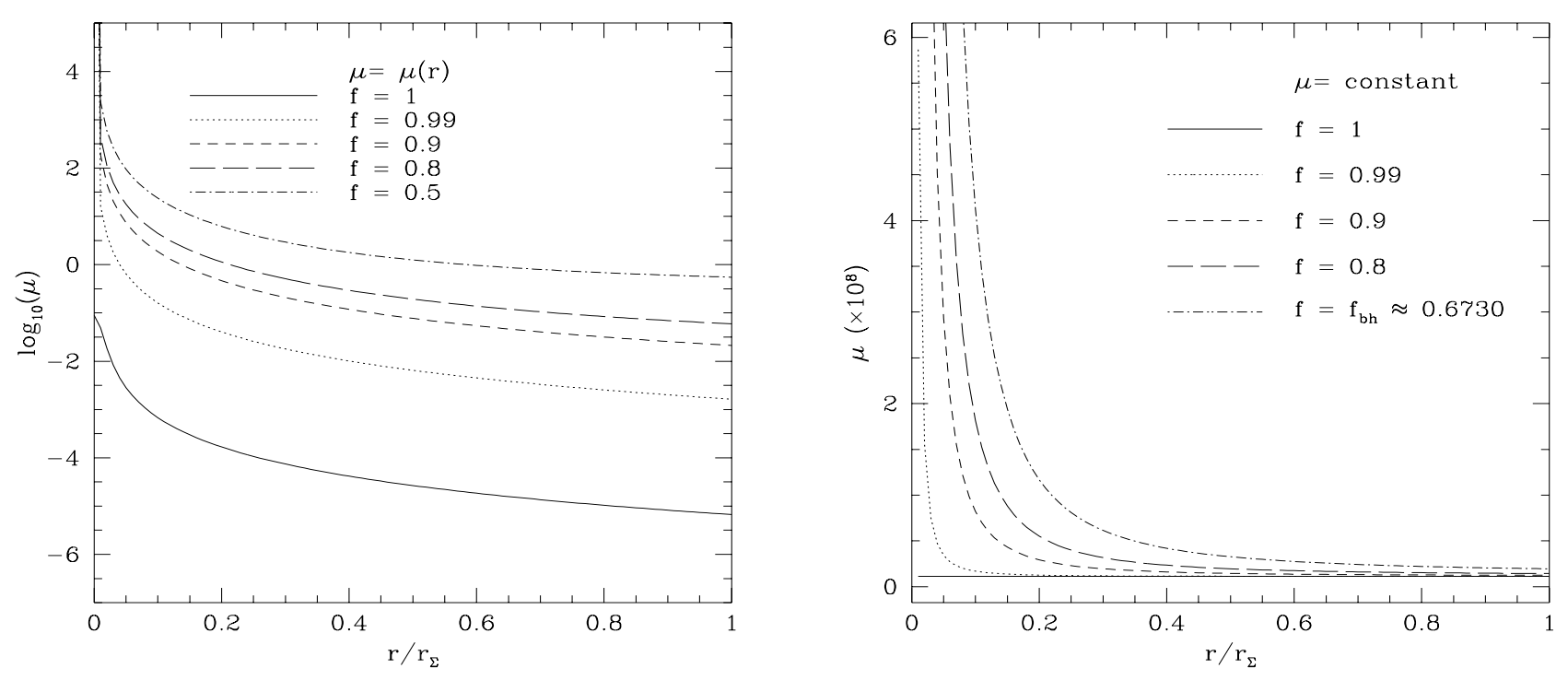

FIG. 4: Density profiles for the model with initial homogeneous and initial inhomogeneous energy density. The radii $r$ and $r_{\Sigma}$ are in units of seconds and the density is in units of $\sec ^{-2}$.
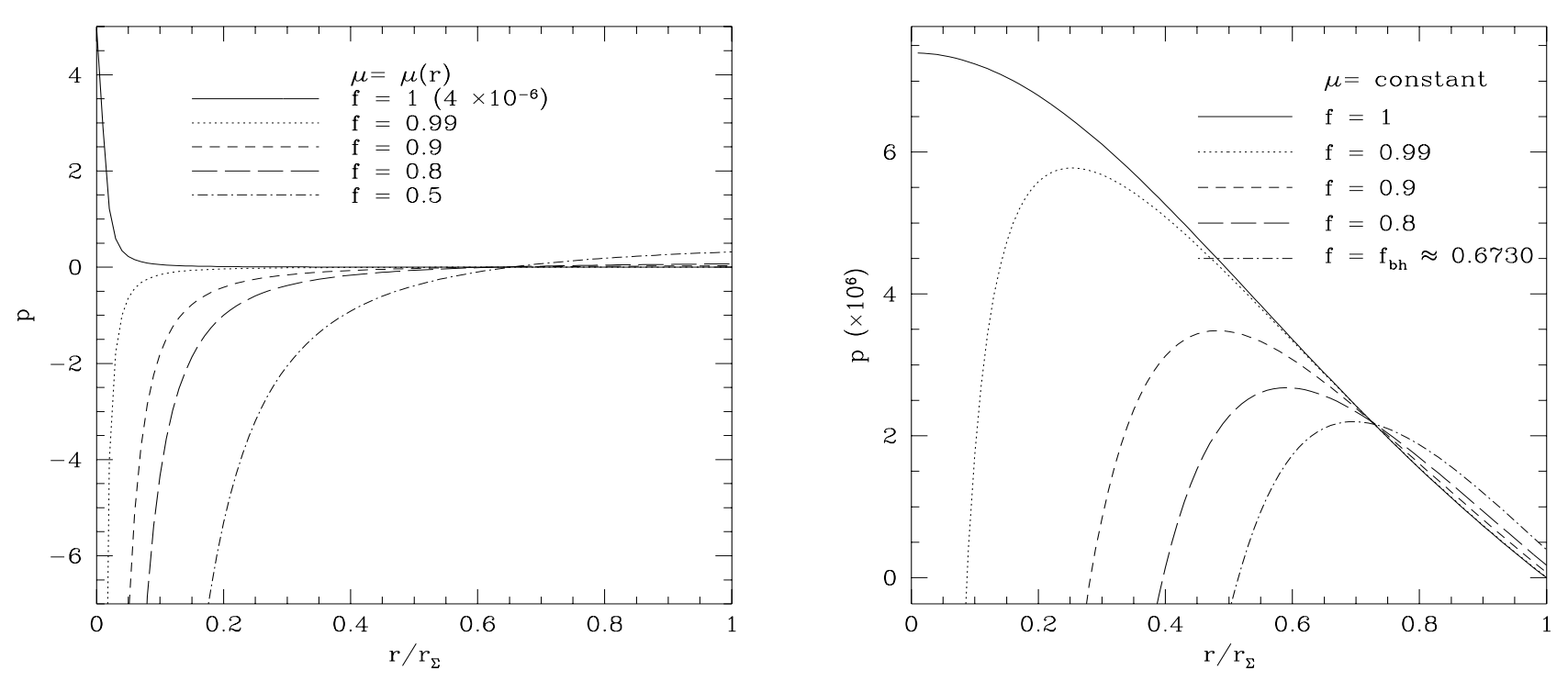

FIG. 5: Radial pressure profiles for the model with initial homogeneous and initial inhomogeneous energy density. The radii $r$ and $r_{\Sigma}$ are in units of seconds and the radial pressure is in units of $\sec ^{-2}$. 

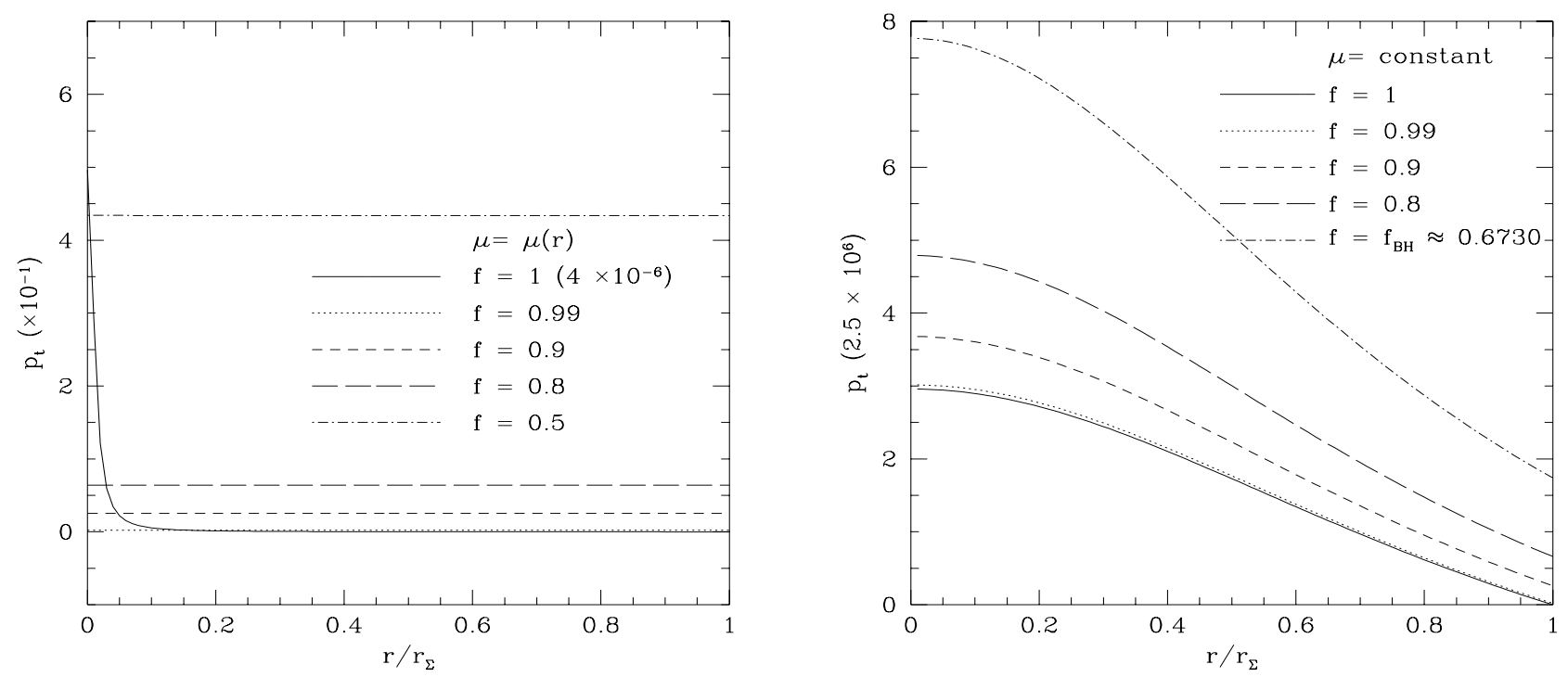

FIG. 6: Tangential pressure profiles for the model with initial homogeneous and initial inhomogeneous energy density. The radii $r$ and $r_{\Sigma}$ are in units of seconds and the tangential pressure is in units of $\mathrm{sec}^{-2}$.
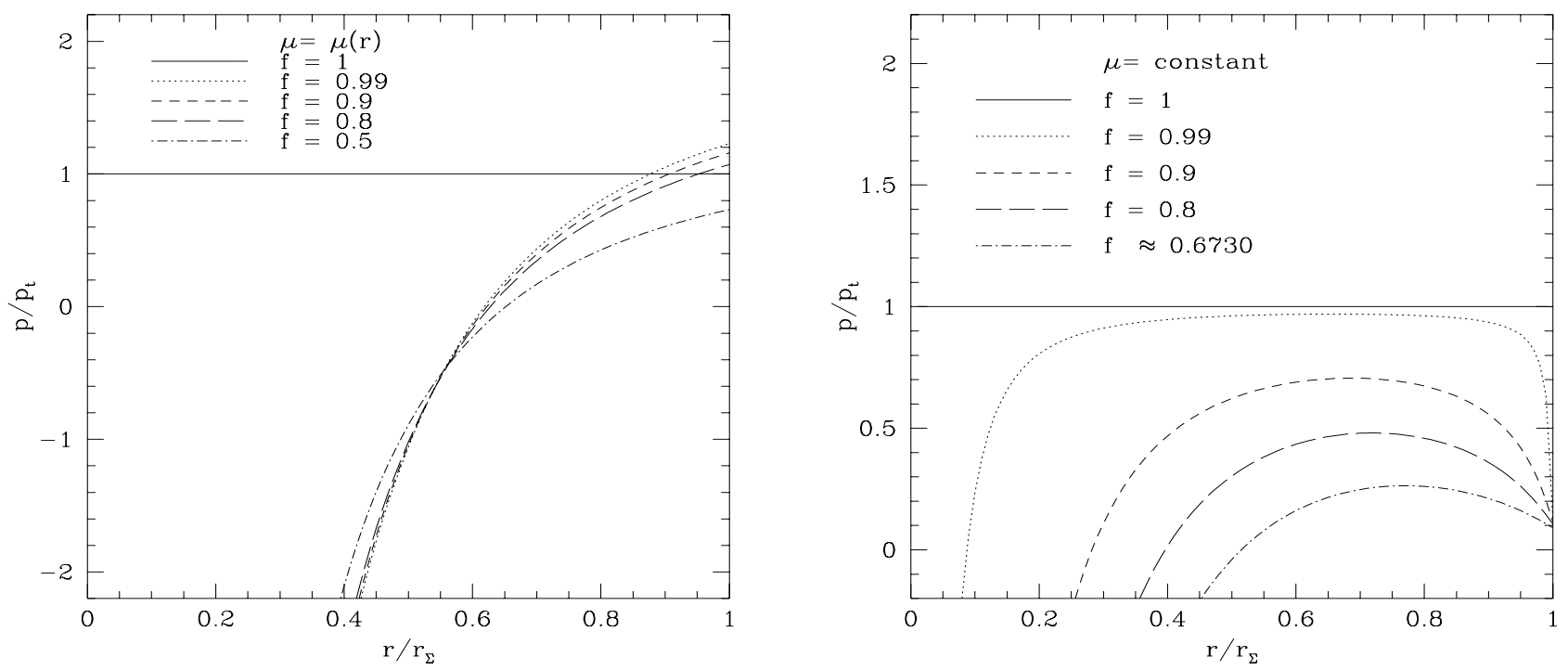

FIG. 7: The ratio of the radial and tangential pressure profiles for the model with initial homogeneous and initial inhomogeneous energy density. The radii $r$ and $r_{\Sigma}$ are in units of seconds and the radial and tangential pressures are in units of $\mathrm{sec}^{-2}$. 

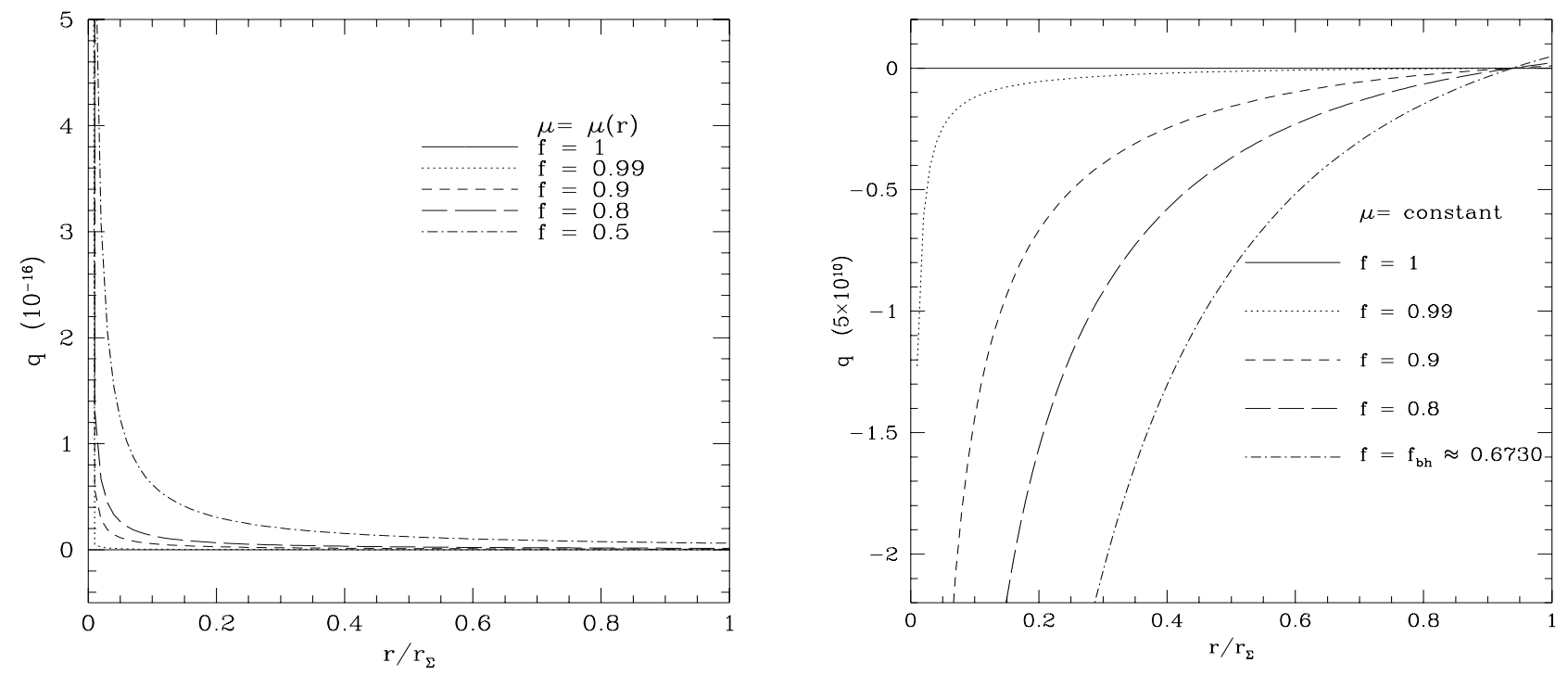

FIG. 8: Heat flux scalar profiles for the model with initial homogeneous and initial inhomogeneous energy density. The radii $r$ and $r_{\Sigma}$ are in units of seconds and the heat flux scalar is in units of $\sec ^{-2}$.

$$
\text { (vi) } \quad 2 p_{t}+\Delta \geq 0
$$

where $\Delta=\sqrt{(\mu+p)^{2}-4 \bar{q}^{2}}$.

From figure 9(i)-(vi) $(\mu=\mu(r))$ we can note that the conditions (56)-(61) are always satisfied for all radii and times. However, in contrast, from the figure 9(i)-(vi) ( $\mu$ = constant) we can conclude that the conditions $(i)$ and $(v i)$ are not satisfied during all the collapse and for any radius. These inequalities are not satisfied for the innermost radii $\left(r \leq 0.2 r_{\Sigma}\right)$ and for the latest stages of the collapse.

\section{PHYSICAL RESULTS}

As in [4] [5] [7] [8], we have calculated several physical quantities, as the total energy entrapped inside the $\Sigma$ surface, the total luminosity perceived by an observer at rest at infinity and the effective adiabatic index, and we have compared them to the respective initial homogeneous ones.

From equation (26) we can write using (30)- $(32)$ that 

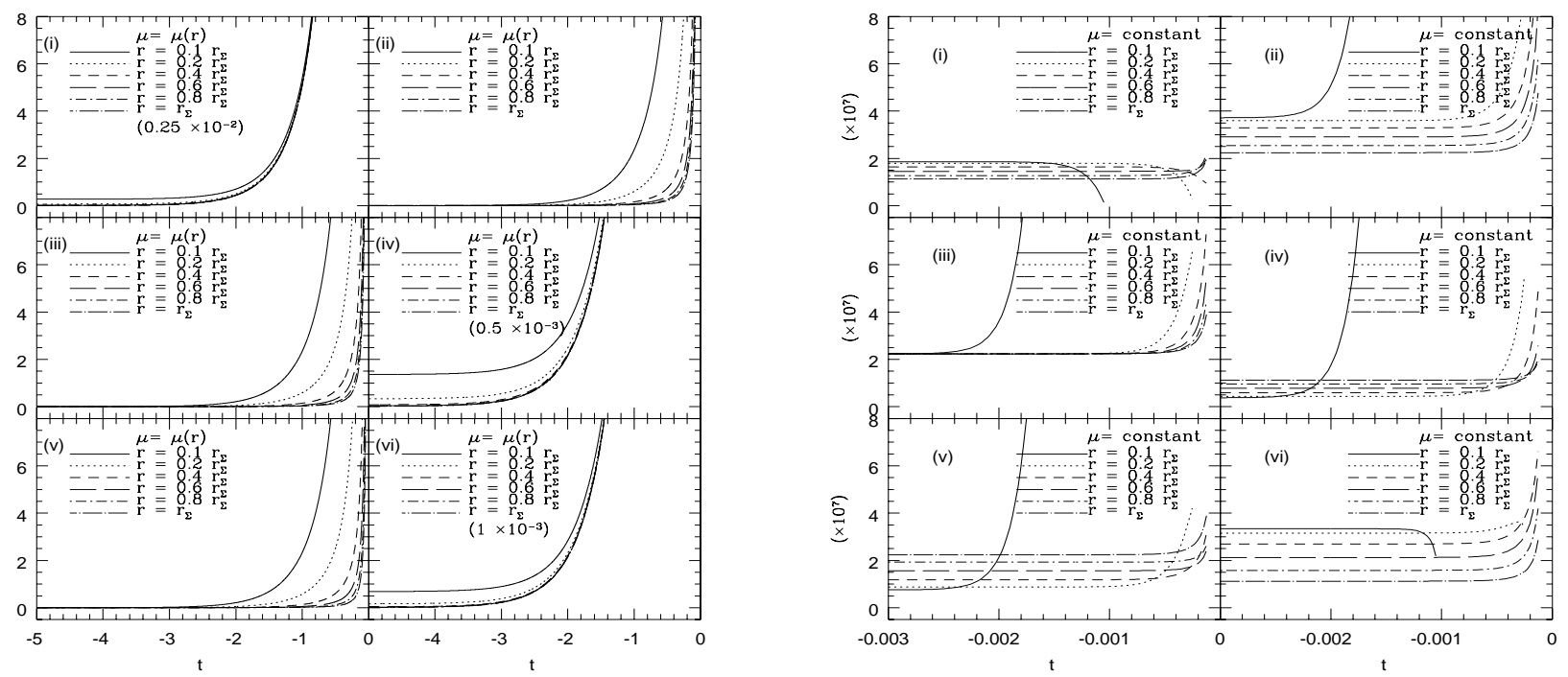

FIG. 9: The energy conditions (56)-(58), for the model with initial inhomogeneous and homogeneous energy density. The time is in units of seconds and all the others quantities are in units of $\sec ^{-2}$.
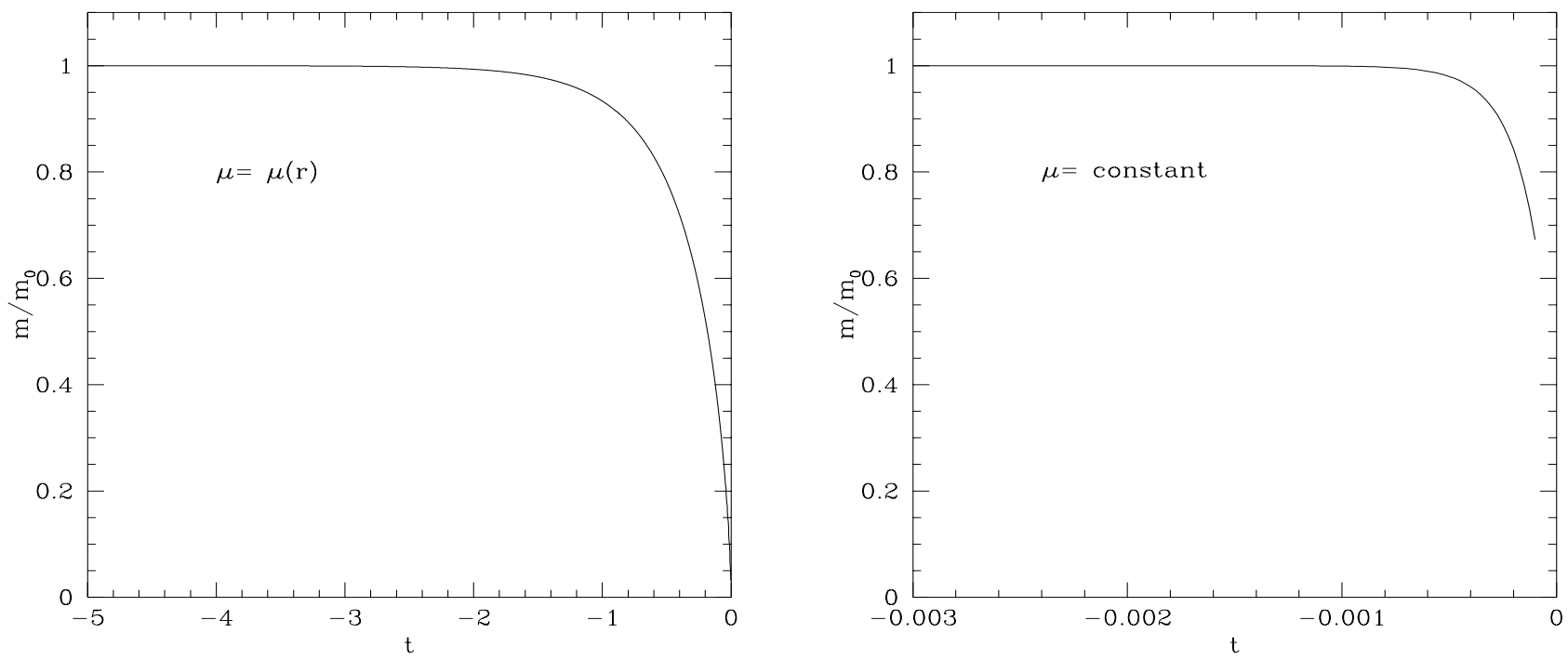

FIG. 10: Time behavior of the total energy entrapped inside the surface $\Sigma$ for the models with initial inhomogeneous and homogeneous energy density. The time, $m$ and $m_{0}$ are in units of seconds. 

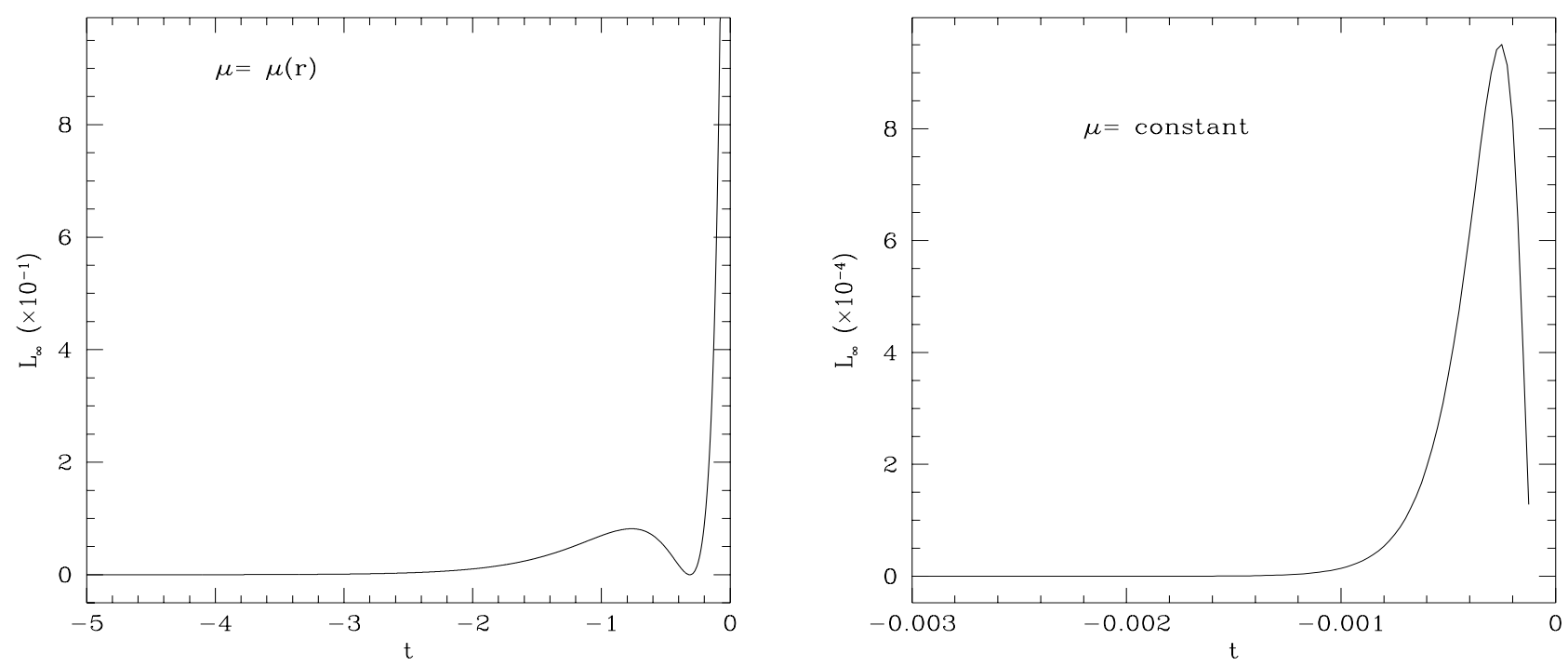

FIG. 11: Time behavior of the luminosity perceived by an observer at rest at infinity for the models with initial inhomogeneous and homogeneous energy density. The time is in units of second and the luminosity is dimensionless.

$$
m=\left[\frac{r^{3} B_{0}^{3}}{2 A_{0}^{2}} f \dot{f}^{2}+\frac{r B_{0}}{2} f\left(1-f^{2}\right)+m_{0} f^{3}\right]_{\Sigma},
$$

where

$$
m_{0}=-\left[r^{2} B_{0}^{\prime}+\frac{r^{3} B_{0}^{\prime 2}}{2 B_{0}}\right]_{\Sigma} .
$$

We can observe from figure 10 that the mass inside $\Sigma$ is different for both models, initial inhomogeneous and homogeneous energy density ones. The initial inhomogeneous star model radiates all its mass during the evolution, in contrast to the initial homogeneous star model where the star radiates about $33 \%$ of its total mass.

Using the equations (28) and (30)-(32) we can write the luminosity of the star as

$$
\begin{aligned}
L_{\infty}= & \frac{\kappa}{2}\left\{p r^{2} B_{0}^{2} f^{2} \times\right. \\
& \left.\times\left[\left(r \frac{B_{0}^{\prime}}{B_{0}}+1\right) f+\left(\frac{r B_{0}}{A_{0}}\right) \dot{f}\right]^{2}\right\}_{\Sigma} .
\end{aligned}
$$



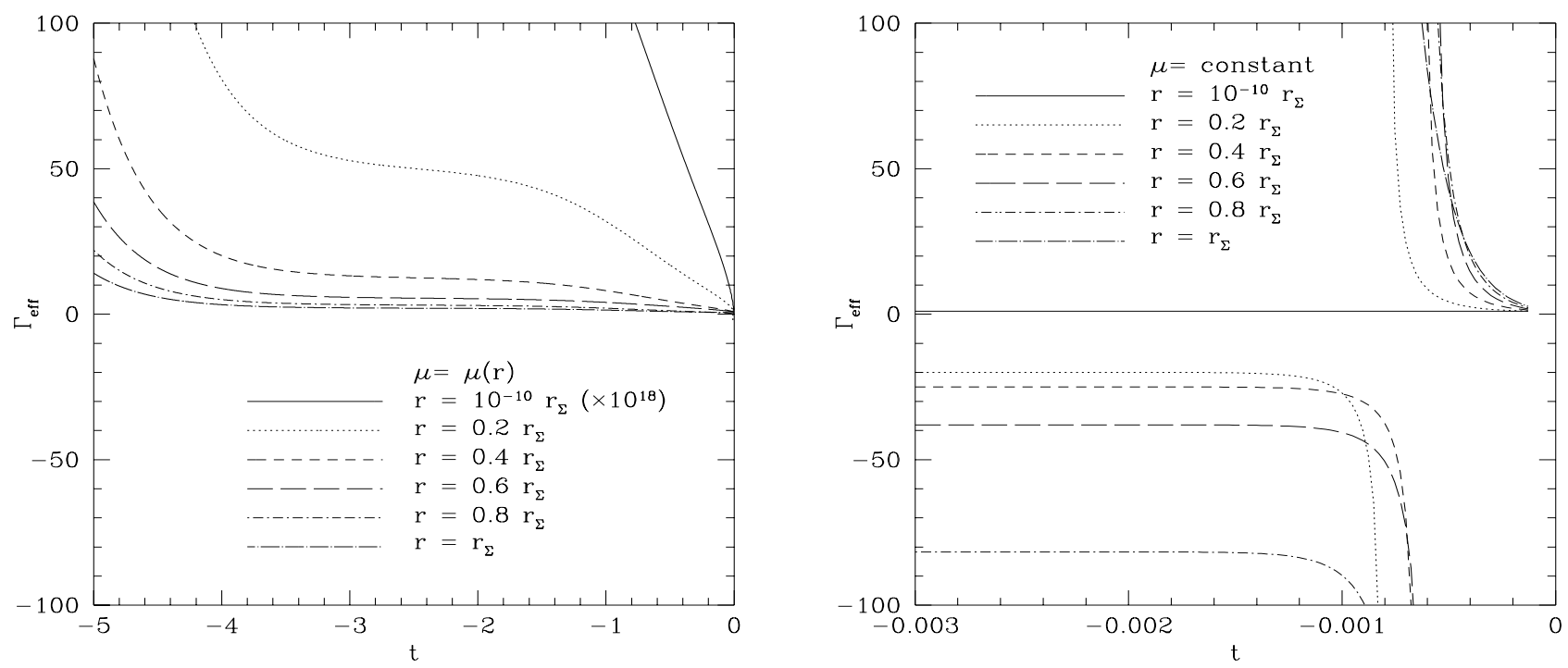

FIG. 12: Time behavior of the effective adiabatic index for the models with initial inhomogeneous and homogeneous energy density. The time is in units of second and $\Gamma_{\text {eff }}$ is dimensionless.

The effective adiabatic index can be calculated using the equations (35)-(36), (44) and (45)-(154). Thus, we can write that

$$
\begin{array}{r}
\Gamma_{\text {eff }}=\left[\frac{\partial(\ln p)}{\partial(\ln \mu)}\right]_{r=c o n s t}=\left(\frac{\dot{p}}{p}\right)\left(\frac{\mu}{\dot{\mu}}\right)= \\
\frac{a B_{0}^{2} f\left[-a f \dot{f}-b\left(1-f^{2}\right)-3 \dot{f}^{2}\right]-4\left(b B_{0}^{2}-A_{0}^{2} / r^{2}\right) \dot{f}}{f^{2} A_{0}^{2} B_{0}^{2} \kappa p_{0}+a B_{0}^{2} f \dot{f}+\left(B_{0}^{2} b-A_{0}^{2} / r^{2}\right)\left(1-f^{2}\right)} \times \\
\frac{f^{2} A_{0}^{2} B_{0}^{2} \kappa \mu_{0}+B_{0}^{2} \dot{f}^{2}+A_{0}^{2}\left(1-f^{2}\right) / r^{2}}{2 B_{0}^{2} \dot{f}\left[-a f \dot{f}-b\left(1-f^{2}\right)-3 \dot{f}^{2}\right]-4 \dot{f} / A_{0}^{2}} .
\end{array}
$$

Comparing the figures for $\Gamma_{\text {eff }}(\mu=\mu(r)$ and $\mu=$ constant $)$ we notice that the time evolution of the effective adiabatic indices are very different graphically. We can note in figure 12 ( $\mu$ = constant) that shortly before the peak of luminosity (see figure 11) there is a large discontinuity in $\Gamma_{\text {eff }}$, in contrast to the initial inhomogeneous star model where there is no discontinuity in $\Gamma_{\text {eff }}$. 


\section{CONCLUSIONS}

A new model is proposed to a collapsing star consisting of an initial inhomogeneous energy density fluid, radial heat flow and outgoing radiation. In previous papers [4]-[8] one of us has introduced a collapse model of an initial homogeneous energy density. In this work we have generalized this previous model by introducing an initial inhomogeneous energy density and we have compared it to the initial homogeneous one.

The behavior of the energy density, pressure, mass, luminosity and the effective adiabatic index was analyzed. We have also compared to the case of a collapsing fluid with initial homogeneous energy density of another previous model [4] [5], for a star with $6 M_{\odot}$.

As we have shown the black hole is never formed because the apparent horizon formation condition is never satisfied. This could be interpreted as the formation of a naked singularity, as Joshi, Dadhich and Maartens [22] have suggested. However this is not the case because the star radiates all its mass before it reaches the singularity at $r=0$ and $t=0$. Not even a marginally naked singularity is formed by the same reason, since in this case the apparent horizon should coincide with the singularity at $r=0$ and $t=0$.

The pressure has negative values although physically this could be considered unreasonable. However, due to the heat flow (the term $\Delta$ ) the energy conditions are always satisfied.

The pressure of the star, at the beginning of the collapse, is isotropic but due to the presence of the shear the pressure becomes more and more anisotropic.

The star radiates all its mass during the collapse and this explains why the apparent horizon never forms. In contrast of the result of this work, the former model radiates about $33 \%$ of the total mass of the star, before the formation of the black hole.

An observer at infinity will see a radial point source radiating exponentially until reaches the time of maximum luminosity and suddenly the star turns off because there is no more mass in order to be radiated. In contrast of the former model with initial homogeneous

energy density [4]-[8] where the luminosity also increases exponentially, reaching a maximum and after it decreases until the formation of the black hole.

The effective adiabatic index, in the initial inhomogeneous energy density model, is always positive without any discontinuity in contrast of the former model where there is a discontinuity around the time of maximum luminosity. In the case of initial homogeneous energy density, the effective adiabatic index has a very unusual behavior because we have 
a non-adiabatic regime in the fluid due to the heat flow. The index becomes negative since the hydrodynamic pressure and the energy density may become negative. Besides, in this case, neither the energy density is the measure of the total energy density of a given piece of matter nor the hydrodynamic pressure the only opposing contraction [23].

\section{ACKNOWLEDGMENTS}

The author (RC) acknowledges the financial support from FAPERJ (no. E26/171.754/2000, E-26/171.533/2002 and E-26/170.951/2006) and from Conselho Nacional de Desenvolvimento Científico e Tecnológico - CNPq - Brazil. The author (GP) also acknowledges the financial support from CAPES.

[1] Bonnor, W.B., de Oliveira, A.K.G. and Santos, N.O. 1989, Phys. Rep. 181, 269

[2] Cahill, M.E. and McVittie G.C. 1970, J. Math. Phys. 11, 1382

[3] Chan, R. 1993, A\&SS 206, 219

[4] Chan, R. 1997, MNRAS 288, 589

[5] Chan, R. 1998, MNRAS 299, 811

[6] Chan, R. 1998, A\&SS 257, 299

[7] Chan, R. 2000, MNRAS 316, 588

[8] Chan, R. 2001, A\&A 368, 325

[9] Chan, R., Int. J. Mod. Phys. D 12, 1131 (2003).

[10] de Oliveira, A.K.G., Santos, N.O. and Kolassis, C.A., 1985, MNRAS 216, 1001

[11] Herrera, L. and Santos, N.O. 1997, Phys. Rep. 286, 53

[12] Israel, W. 1966a, Nuovo Cimento 44B, 1

[13] Israel, W. 1966b, Nuovo Cimento 48B, 463

[14] Kolassis, C.A., Santos, N.O. and Tsoubelis, D. 1988, Class. Quantum Grav. 5, 1329

[15] Santos, N.O. 1985, MNRAS 216, 403

[16] Vaidya, P.C. 1953, Nature 171, 260

[17] Wagh, S.M., Govender, M., Govinder, K.S., Maharaj, S.D., Muktibodh, P.S. and Moodley, M. 2001, Class. Quantum Grav. 18, 2147

[18] Woosley, S.E. and Phillips, M.M. 1988, Science 240, 750 
[19] Nogueira, P.C. and Chan, R., Int. J. Mod. Phys. D 13, 1727 (2004).

[20] Pinheiro, G. and Chan, R. 2008, Gen. Rel. Grav. 40, 2149 (2008).

[21] Pinheiro, G. and Chan, R., Int. J. Mod. Phys. D, in press (2010).

[22] Joshi, P.S., Dadhich, N. and Maartens, R., Phys. Rev. D 65, 101501 (2002).

[23] Barreto, W., Herrera, L. and Santos, N.O., Astrophys. Spa. Sci. 187, 271 (1992).

[24] Misthry, S.S., Maharaj, S.D. and Leach, P.G.L., Math. Meth. Appl. Sci. 31, 363 (2008).

[25] Herrera, L., Di Prisco, A., Fuenmayor, E. and Troconis, O., Int. J. Mod. Phys. D 18, 129 (2009).

[26] Naidu, N.F., Govender, M. and Govinder, K.S., Int. J. Mod. Phys. D 15, 1053 (2006).

[27] Di Prisco, A., Herrera, L., Le Denmat, G., MacCallum, M.A.H., and Santos, N.O., Phys. Rev. D 76, 6, 064017 (2007).

[28] Sharif, M. and Khadija I., Mod. Phy. Lett. A 24, 1533 (2009).

[29] Goldman, S.P., Astrophys. J. 226, 1079 (1978).

[30] Herrera, L., Di Prisco, A., Hernandez-Pastora, J., Santos, N.O., Phys. Lett. A 237, 113 (1998).

[31] Herrera, L., Di Prisco, A., Gen. Rel. Grav. 31, 301, (1999). 